\title{
Outcomes of Extracorporeal Membrane Oxygenation in Acute Respiratory Distress Syndrome due to COVID-19, lessons learned from the first wave of COVID 19
}

\author{
Cameron Blazoski ${ }^{1}$, Michael Baram ${ }^{1}$, and Hitoshi Hirose ${ }^{1}$ \\ ${ }^{1}$ Thomas Jefferson University Hospital
}

January 10, 2021

\begin{abstract}
Introduction: Extracorporeal membrane oxygenation (ECMO) has been used as a refractory treatment for acute respiratory distress syndrome (ARDS) due to COVID-19, but there has been little evidence of its efficacy. We conducted this study to share our experience using ECMO as a bridge to recovery for ARDS due to COVID-19. Methods: All adult patients who were placed on ECMO for ARDS due to COVID -19 between April 2020 and June 2020 (during the first wave of COVID-19) were identified. The clinical characteristics and outcomes of these patients were analyzed with a specific focus on the differences between patients who survived to hospital discharge and those who did not. Results: 20 COVID-19 patients were included in this study. All patients were placed on veno-veno ECMO. Comparing between survivors and non-survivors, older age was associated with hospital mortality $(\mathrm{p}=0.02)$. The following complications were observed: renal failure requiring renal replacement therapy $(35 \%, \mathrm{n}=7)$, bacteremia during ECMO $(20 \%, \mathrm{n}=4)$, coinfection with bacterial pneumonia $(15 \%, \mathrm{n}=3)$, cannula site bleeding $(15 \%, n=3)$, stroke $(10 \%, n=2)$, gastrointestinal bleeding $(10 \%, n=2)$, and liver failure $(5 \%, n=1)$. The complications associated with patient mortality were culture positive septic shock $(\mathrm{p}=0.01)$, culture-negative systemic inflammatory response syndrome $(\mathrm{p}=0.01)$, and renal failure $(\mathrm{p}=0.01)$. The causes of death were septic shock $(44 \%, \mathrm{n}=4)$, culture-negative systemic inflammatory response syndrome $(44 \%, \mathrm{n}=4)$, and stroke $(11 \%, \mathrm{n}=1)$. Conclusions: Based on our experience, ECMO can improve refractory ARDS due to COVID-19 in select patients. Proper control of bacterial infections during COVID-19 immunomodulation therapy may be critical to improving survival.
\end{abstract}

Outcomes of Extracorporeal Membrane Oxygenation in Acute Respiratory Distress Syndrome due to COVID-19, lessons learned from the first wave of COVID 19.

Cameron Blazoski, BA ${ }^{1}$, Michael Bram, $\mathrm{MD}^{2}$, Hitoshi Hirose $\mathrm{MD}^{1}$.

Department of Surgery, Thomas Jefferson University, Philadelphia, PA

Department of Medicine, Pulmonary and Critical Care, Thomas Jefferson University, Philadelphia, PA

Corresponding author: Hitoshi Hirose, MD, PhD.

Department of Surgery, Thomas Jefferson University, Philadelphia, PA.

1025 Walnut St, Ste 605, Philadelphia, PA 19107

Tel: 215-955-6996; Fax: 215-955-6010; E-mail: genex@nifty.com

Conflict of Interests: None declared

Financial support: None

Word count :1896 


\begin{abstract}
Introduction: Extracorporeal membrane oxygenation (ECMO) has been used as a refractory treatment for acute respiratory distress syndrome (ARDS) due to COVID-19, but there has been little evidence of its efficacy. We conducted this study to share our experience using ECMO as a bridge to recovery for ARDS due to COVID-19.
\end{abstract}

Methods: All adult patients who were placed on ECMO for ARDS due to COVID -19 between April 2020 and June 2020 (during the first wave of COVID-19) were identified. The clinical characteristics and outcomes of these patients were analyzed with a specific focus on the differences between patients who survived to hospital discharge and those who did not.

Results: 20 COVID-19 patients were included in this study. All patients were placed on veno-veno ECMO. Comparing between survivors and non-survivors, older age was associated with hospital mortality $(\mathrm{p}=0.02)$. The following complications were observed: renal failure requiring renal replacement therapy $(35 \%, \mathrm{n}=7)$, bacteremia during ECMO $(20 \%, \mathrm{n}=4)$, coinfection with bacterial pneumonia $(15 \%, \mathrm{n}=3)$, cannula site bleeding $(15 \%, \mathrm{n}=3)$, stroke $(10 \%, \mathrm{n}=2)$, gastrointestinal bleeding $(10 \%, \mathrm{n}=2)$, and liver failure $(5 \%, \mathrm{n}=1)$. The complications associated with patient mortality were culture positive septic shock $(\mathrm{p}=0.01)$, culture-negative systemic inflammatory response syndrome $(\mathrm{p}=0.01)$, and renal failure $(\mathrm{p}=0.01)$. The causes of death were septic shock $(44 \%, n=4)$, culture-negative systemic inflammatory response syndrome $(44 \%, n=4)$, and stroke $(11 \%, \mathrm{n}=1)$.

Conclusions: Based on our experience, ECMO can improve refractory ARDS due to COVID-19 in select patients. Proper control of bacterial infections during COVID-19 immunomodulation therapy may be critical to improving survival.

(Word count of abstract: 250)

\title{
Introduction
}

Since its outbreak in 2019, there have been over 50 million cases of coronavirus disease 2019 (COVID-19) and 1.25 million recorded deaths.(1) While most patients experience mild to moderate symptoms, COVID-19 can progress to acute respiratory distress syndrome (ARDS), a rapidly progressive inflammatory syndrome that impairs oxygen transport in the lungs. $(2,3)$ The pulmonary injury in ARDS due to COVID-19 has been shown to resemble ARDS unrelated to COVID-19, and even with mechanical support, ARDS is associated with a significant mortality among COVID-19 patients. $(2,4,5)$

The high mortality rate of ARDS due to COVID-19 increased the demand for other treatment options, and the use of extracorporeal membrane oxygenation (ECMO) was encouraged for select cases of refractory ARDS with severe hypoxemia.(5-8) ECMO is a temporary form of mechanical cardiopulmonary support for patients with severe cardiac and/or respiratory shock. First clinically used in 1972, ECMO's use has exponentially increased in the past two decades.(9-11) While its efficacy in lowering mortality rates is still debated, ECMO is now a common treatment for patients with refractory ARDS.(11-14)

Despite some recent publications,(15-17) there remains a lack of evidence documenting the overall efficacy of ECMO in treating ARDS due to COVID-19. The purpose of this paper is to share our experience using ECMO as a bridge to recovery for patients with ARDS due to COVID-19 during the first wave of the COVID-19 pandemic in our area.

\section{Methods}

Adult patients positive for COVID-19 who underwent ECMO at our institution from April 1, 2020 to June 11, 2020 were included in this study. Patients were identified by an IRB-approved, prospectively maintained ECMO database (IRB approval \# 11D.185). The data from these patients was retrospectively extracted and details were further studied by reviewing medical records. Inclusion criteria included a positive COVID-19 test and a diagnosis of ARDS. ECMO placement was determined by a multidisciplinary team that included a cardiac surgeon, a pulmonary critical care physician, and a cardiovascular intensivist. 
The indications for ECMO placement were the same as our previous paper,(18) and contraindications for ECMO placement in treating COVID-19 patients are listed in Table 1. The exclusion criteria may be more restricted than in non-COVID-19 patients, due to the limitations of resources during this pandemic.

The primary mode of ECMO in COVID-19 ARDS was veno-venous ECMO (VV-ECMO) using the femoral and internal jugular veins (Figure 1). All cannulation was performed in the ICU without transport to either the operating room or catheterization lab unless an issue occurred during bedside cannulation. Since Avalon dual lumen ECMO cannula placement always requires fluoroscopy and echocardiography, which requires additional personnel including radiology technicians and an echocardiography technician, the utilization of the Avalon cannula was discouraged.(18) Veno-arterial ECMO (VA-ECMO) should be reserved for only those who had severe but reversible cardiac dysfunction, such as COVID-19 related myocarditis. ECMO assisted cardiopulmonary resuscitation (ECPR) for patients with COVID-19 was discouraged due to its known poor outcomes.

Due to the COVID-19 pandemic, we no longer offer a mobile ECMO program outside of our institutional network due to the concern of exposure of required personnel including the ECMO surgeon, perfusionist, and transfer nurses at the local site. Instead of activating mobile ECMO cannulation teams, we encouraged local cardiac surgeons to place ECMO at their institutions and then transport the patient to our facility.

The general management of ECMO has been described in one of our prior papers.(19) Briefly, after placement of ECMO, the ventilator was set to the ARDSnet protocol. The typical setting was pressure controlled ventilation, rate 15, PEEP 15, delta P 15 until recovery of the respiratory function. Paralytics were discontinued within 24 hours of ECMO initiation, unless respiratory function was deetiolated. Sedatives were used if a RAS score was negative 1-2. Blood pressure was maintained at least mean arterial pressure of $60 \mathrm{~mm} \mathrm{Hg}$ with vasopressors and/or fluid as appropriate. A heparin drip was started once PTT fell below 50 seconds after cannulation and maintained at an anti-Xa level of 0.3-0.5. If bleeding complications were observed, the anticoagulation was held and then restarted at a lower anti-Xa goal of 0.1-0.3.

Timing of the decannulation was determined by chest x-ray findings and lung mechanics. Before decannulation, the sweep gas was discontinued for at least 24 hours to ensure the lungs were able to exchange oxygen and carbon dioxide appropriately. For COVID-19 cases, we encourage bed-side decannulation rather than transporting to operating room to limit exposure to COVID-19.

The primary endpoints of this study were ECMO survival and hospital survival. ECMO survival was defined by withdrawal of care or death within 24 hours of decannulation. All patients who survived to hospital discharge were classified as "Survivors" and all patients who did not survive to hospital discharge were classified as "Non-Survivors." The baseline characteristics, clinical characteristics, and outcomes were calculated and compared between the two groups.

Data was expressed as the number with percentage, mean +/- standard deviation, or median (quantile) as appropriate. Two groups were compared using chi-squared tests for categorical variables and standard t-tests for continuous variables as appropriate. Significance was accepted at a P-value less than 0.05.

\section{Results}

During this study period, 20 patients with ARDS positive for COVID-19 underwent ECMO placement. All patients were placed on veno-veno ECMO, and the mean length of ECMO placement was 14.0 days. The average length of symptoms prior to ECMO placement was 11.4 days, with the average patient spending 10.3 days in the hospital prior to ECMO placement. The average time spent on a ventilator prior to ECMO placement was 147 hours. $75 \%$ of patients $(n=15)$ were transferred from another institution to our hospital, and $60 \%$ of patients $(\mathrm{n}=12)$ had ECMO initiated at another institution prior to transfer. PreECMO characteristics and patient demographics are displayed in Table 2. Patients were also treated with different therapies prior to starting ECMO placement. Therapy was administered in the form of steroids $(65 \%, \mathrm{n}=13)$, interleukin-6 inhibitors $(55 \%, \mathrm{n}=11)$, remdesivir $(20 \%, \mathrm{n}=4)$, and plasma $(15 \%$, $\mathrm{n}=3)$. None of the treatment therapies were associated with better or worse mortality rates. The types of treatment and 
their statistics are displayed in Table 3.

The ECMO survival rate was (15/20), and the survival rate to hospital discharge was $55 \%(11 / 20)$. 11 patients were labeled as Survivors and 9 as Non-Survivors based on their survival to hospital discharge. The only baseline characteristic that was statistically different between the two groups was age, as Non-Survivors were significantly older than Survivors (58.4 vs. 49.6, $\mathrm{p}=0.02$ ).

The causes of death were septic shock $(44 \%, \mathrm{n}=4)$, culture-negative systemic inflammatory response syndrome (SIRS) $(44 \%, \mathrm{n}=4)$, and stroke $(11 \%, \mathrm{n}=1)$. The most common complication observed was renal failure requiring renal replacement therapy $(35 \%, \mathrm{n}=7)$. Complications that were associated with patient mortality between the two groups were blood culture positive sepsis $(\mathrm{p}=0.01)$, culture-negative systemic inflammatory response syndrome (SIRS) $(\mathrm{p}=0.01)$, cannula site bleeding $(\mathrm{p}=0.04)$, and renal failure $(\mathrm{p}=0.01)$. The complications are displayed in Table 4.

\section{Discussion}

The primary finding of this study is that among the 20 COVID-positive patients that we treated with ECMO, the survival rate to hospital discharge was $55 \%$. Also, our results suggest that proper patient selection and control of bacterial infections prior to and/or during ECMO placement may be key to improving survival, as culture-positive septic shock and culture-negative SIRS were the main causes of death and were only observed in patients who failed to survive to hospital discharge.

The treatment of ARDS with ECMO remains disputed, even though its use in treating ARDS has increased in the past decade. $(11,12)$ While the exact mortality rate of treating ARDS with ECMO varies by research study, it is generally accepted to range between $34-39 \% .(11,13,14)$ Thus, it is generally recognized that ECMO should be primarily used for refractory cases of ARDS, in which a patient remains severely hypoxic despite aggressive treatment.(12)

Interestingly, our study and others on COVID-19 have found that the mortality rates of treating ARDS due to COVID-19 with ECMO are similar to the previously reported mortality rates of treating non-COVID ARDS. Recently, there have been a few articles published that specifically investigated the use of ECMO in treating ARDS due to COVID-19. The largest of these studies was conducted by Barbaro et al., who drew from an Extracorporeal Life Support Organization (ELSO) registry to analyze the outcomes of 1035 COVID-positive patients who were treated with ECMO.(15) The researchers found that among these patients, the 90-day post-ECMO mortality rate was $37.4 \%$. The study included data from 213 different hospitals and included patients treated from January $16^{\text {th }}$ to May $1^{\text {st }}$ of 2020 . Another study that more closely resembles our own is from Schmidt et al, who documented the outcomes of 83 COVID-positive patients who were treated at their hospital.(16) They discovered that the 60-day mortality of these patients was $31 \%$. Compared to these studies, our patients had a slightly higher mortality rate of $45 \%$; however, it should be noted that our sample size was significantly smaller than either of these two articles.

Based on this current research on the use of ECMO in ARDS due to COVID-19, the mortality rate appears to be anywhere between 31-45\%. This mortality rate is similar to the 34-39\% mortality rate in treating non-COVID ARDS with ECMO in select patients. Therefore, it is possible that ECMO is just as effective at treating ARDS due to COVID-19 as it is at treating ARDS due to non-COVID-19 etiologies if appropriate patient selection was applied. For example, none of the patients in our study had cardiac dysfunction. This is because our selection committee considered COVID-19 patients with cardiac dysfunction and ARDS to have multiorgan failure, which was a contraindication for ECMO placement. While there needs to be far more research done on this topic to definitively state that ECMO is effective in treating COVID-19, it is possible that it is an effective treatment option for refractory cases of ARDS due to COVID-19.

Sepsis and SIRS were the causes of death in all but one patient in our study, suggesting that bacterial infections during ECMO placement may be a significant factor in mortality rates. While immunomodulation therapy has been shown to decrease the mortality rate of COVID-19,(20,21) it has also been associated with an increased infection rate. For example, one study demonstrated that $13 \%$ of patients treated with 
tocilizumab were diagnosed with new infections, compared to only $4 \%$ of patients treated solely with the standard of care.(21) There should be particular attention to preventing, monitoring for, and responding early to bacterial infections in COVID-19 patients placed on ECMO.

During the first wave of COVID-19 in Pennsylvania, the highest number of daily COVID-19 cases occurred on April $8^{\text {th }}$ with 2,059 cases. By the end of our study period on June $11^{\text {th }}$, the daily number of cases fell to 680, which was one of the lowest number of daily cases in Pennsylvania since the beginning of the pandemic. Our study beings on April $1^{\text {st }}$ and ends on June $11^{\text {th }}$, which fairly accurately represents the beginning and end of the first wave of COVID-19 in Pennsylvania. Beginning in late October, Pennsylvania entered a second wave, with daily cases greatly exceeding those of the first wave. Future studies of the second wave of COVID-19 will differ from this study, for multiple reasons, including changes in pre-ECMO immunomodulation treatment. During the second wave, interleukin inhibitors are no longer recommended and steroids are more widely used.

Our study is limited by its small sample size and being based in one hospital center. It is also possible that there was selection bias in this study, even though ECMO placement was determined by a multidisciplinary team of physicians.

Despite its limitations, this study provides extensive data on 20 patients with ARDS due to COVID-19 who were treated with ECMO. While we cannot extrapolate from a sample size of 20 patients, we hope our evidence can complement other studies and contribute to meaningful meta-analyses and statistical analyses. Cases of ARDS due to COVID-19 will continue in the coming months and years, and we hope that our analysis contributes to the growing research on how to treat this deadly disease.

\title{
Conclusion
}

Based on our results, we conclude that ECMO placement can improve refractory cases of ARDS due to COVID-19. More research is needed to better understand the true efficacy of ECMO in treating COVID-19 and the mortality rate associated with it.

Table 1 : Contraindications for ECMO in COVID 19.

\author{
Standard contraindications \\ Age $>70$ \\ Body mass index $>45$ with high risk of vascular access \\ Mechanical ventilation $>7$ days \\ Multiorgan failure \\ End stage liver disease \\ Irreversible neurological damage \\ Contraindications of anticoagulation \\ Cardiac arrest without ROSC \\ Relative contraindications \\ Age $>65$ \\ Body mass index $>35$ \\ Mechanical ventilation $>5$ days \\ Active bacterial blood stream infection \\ Severe COPD Cirrhosis Chronic heart failure \\ Inability of access neuro status \\ High lactate related to low perfusion status \\ Limited activity at home \\ No family or appropriate power of attorney Outside of institutional network \\ Considering Veno-arterial cannulation \\ Cardiac arrest with ROSC \\ Poor left or right ventricular function
}




\section{Standard contraindications}

Known pulmonary hypertension

Table 2 : Demographics and baseline characteristics of studied patients. Data is expressed with number (percentage) or mean \pm standard deviation.

\begin{tabular}{llllc}
\hline Category & All patients & Survivors & Non-Survivors & \\
\hline Number of patients & $\mathrm{n}=20$ & $\mathrm{n}=11$ & $\mathrm{n}=9$ & P-value \\
Characteristics & & & & \\
Age (years) & $54 \pm 8.7$ & $50 \pm 8.5$ & $58 \pm 6.4$ & $\mathbf{0 . 0 2 0}$ \\
Male & $12(60 \%)$ & $7(64 \%)$ & $5(56 \%)$ & 0.713 \\
Body surface area $\left(\mathrm{cm}^{2}\right)$ & $2.1 \pm 0.3$ & $2.1 \pm 0.3$ & $2.0 \pm 0.3$ & 0.259 \\
Body mass index & $35 \pm 7$ & $38 \pm 7$ & $32 \pm 7$ & 0.057 \\
Underlying Conditions & & & & \\
Pre-ECMO positive blood culture & $5(25 \%)$ & $3(27 \%)$ & $2(22 \%)$ & 0.795 \\
Pre-ECMO time on ventilator (hrs) & $148 \pm 255$ & $206 \pm 344$ & $76 \pm 68$ & 0.280 \\
Smoking & $4(20 \%)$ & $3(27 \%)$ & $1(11 \%)$ & 0.369 \\
Chronic lung disease & $2(10 \%)$ & $0(0 \%)$ & $2(22 \%)$ & 0.099 \\
Diabetes & $6(30 \%)$ & $2(18 \%)$ & $4(44 \%)$ & 0.202 \\
Liver failure & $1(5 \%)$ & $0(0 \%)$ & $1(11 \%)$ & 0.257 \\
Chronic immunosuppression & $1(5 \%)$ & $0(0 \%)$ & $1(11 \%)$ & 0.257 \\
Pre-ECMO acute renal injury & $6(30 \%)$ & $2(18 \%)$ & $4(44 \%)$ & 0.202 \\
Pre-ECMO Vital signs & & & & \\
Length of symptoms (days) & $11.4 \pm 6.5$ & $9.9 \pm 4.6$ & $13.5 \pm 8.4$ & 0.237 \\
Temperature ( ${ }^{\circ}$ F) & $99.4 \pm 1.4$ & $99.7 \pm 1.6$ & $99.0 \pm 1.1$ & 0.281 \\
Heart Rate & $102 \pm 24$ & $111 \pm 23$ & $91 \pm 21$ & 0.064 \\
Respiratory rate & $28 \pm 5.3$ & $27 \pm 3.9$ & $29 \pm 6.7$ & 0.330 \\
Mean arterial pressure (mm Hg) & $85 \pm 17$ & $87 \pm 16$ & $84 \pm 20$ & 0.759 \\
FiO2 (\%) & $93 \pm 14$ & $90 \pm 15$ & $96 \pm 13$ & 0.390 \\
PEEP (cm) & $16 \pm 4.8$ & $17 \pm 5.6$ & $15 \pm 3.6$ & 0.345 \\
Other & & & & \\
Pre-ECMO days in hospital (days) & $10 \pm 13$ & $12 \pm 16$ & $8.8 \pm 7.7$ & 0.654 \\
Transfer from outside hospital & $15(75 \%)$ & $8(73 \%)$ & $7(78 \%)$ & 0.795 \\
ECMO initiated outside hospital & $12(60 \%)$ & $6(55 \%)$ & $6(67 \%)$ & 0.582 \\
Length on ECMO (days) & $14 \pm 9.6$ & $11 \pm 6.2$ & $17 \pm 12$ & 0.183 \\
\hline
\end{tabular}

Table 3 : Treatment modalities provided pre-ECMO placement. Data is expressed with number (percentage).

\begin{tabular}{lllll}
\hline Category & All patients & Survivors & Non-Survivors & \\
\hline Number of patients & $\mathrm{n}=20$ & $\mathrm{n}=11$ & $\mathrm{n}=9$ & P-values \\
Pre-ECMO Treatment & & & & \\
Steroids & $8(40 \%)$ & $5(45 \%)$ & $3(33 \%)$ & 0.582 \\
Interleukin inhibitor & $11(55 \%)$ & $7(64 \%)$ & $4(44 \%)$ & 0.391 \\
Remdesivir & $4(20 \%)$ & $3(27 \%)$ & $1(11 \%)$ & 0.369 \\
Plasma & $3(15 \%)$ & $2(18 \%)$ & $1(11 \%)$ & 0.660 \\
\hline
\end{tabular}


Table 4 : Rates of ECMO complications. Data is expressed with number (percentage)

\begin{tabular}{lllll}
\hline Category & All patients & Survivors & Non-Survivors & \\
\hline $\begin{array}{l}\text { Number of patients } \\
\text { Complications }\end{array}$ & $\mathrm{n}=20$ & $\mathrm{n}=11$ & $\mathrm{n}=9$ & P-values \\
Renal failure & $7(35 \%)$ & $1(9.1 \%)$ & $6(67 \%)$ & $\mathbf{0 . 0 0 7}$ \\
Liver failure & $1(5 \%)$ & $0(0 \%)$ & $1(11 \%)$ & 0.257 \\
Stroke & $2(10 \%)$ & $0(0 \%)$ & $2(22 \%)$ & 0.099 \\
Intracranial bleed & $1(5 \%)$ & $0(0 \%)$ & $1(11 \%)$ & 0.257 \\
Bacterial pneumonia & $3(15 \%)$ & $2(18 \%)$ & $1(11 \%)$ & 0.660 \\
Cannula site bleed & $3(15 \%)$ & $0(0 \%)$ & $3(33 \%)$ & $\mathbf{0 . 0 3 7}$ \\
Gastrointestinal bleed & $2(10 \%)$ & $0(0 \%)$ & $2(22 \%)$ & 0.099 \\
New infection during ECMO & $9(45 \%)$ & $3(27 \%)$ & $6(67 \%)$ & 0.078 \\
Culture-positive sepsis & $5(25 \%)$ & $1(9.1 \%)$ & $4(44 \%)$ & 0.069 \\
Septic shock & $4(20 \%)$ & $0(0 \%)$ & $4(44 \%)$ & $\mathbf{0 . 0 1 3}$ \\
Systemic inflammatory response & $4(20 \%)$ & $0(0 \%)$ & $4(44 \%)$ & $\mathbf{0 . 0 1 3}$ \\
\hline
\end{tabular}

\section{Legends of Figure}

Figure 1 : Typical veno-venous cannulation in COVID 19 case.

\section{REFERENCES}

1. WHO Coronavirus Disease (COVID-19) Dashboard [Internet]. [cited 2020 Nov 12]. Available from: https://covid19.who.int

2. Yang X, Yu Y, Xu J, Shu H, Xia J, Liu H, et al. Clinical course and outcomes of critically ill patients with SARS-CoV-2 pneumonia in Wuhan, China: a single-centered, retrospective, observational study. The Lancet Respiratory Medicine. 2020 May;8(5):475-81.

3. Gibson PG, Qin L, Puah SH. COVID-19 acute respiratory distress syndrome (ARDS): clinical features and differences from typical pre-COVID-19 ARDS. Med J Aust [Internet]. 2020 Jun 22 [cited 2020 Nov 12]; Available from: https://www.ncbi.nlm.nih.gov/pmc/articles/PMC7361309/

4. Grasselli G, Tonetti T, Protti A, Langer T, Girardis M, Bellani G, et al. Pathophysiology of COVID-19associated acute respiratory distress syndrome: a multicentre prospective observational study. The Lancet Respiratory Medicine. 2020 Aug;S2213260020303702.

5. Bartlett RH, Ogino MT, Brodie D, McMullan DM, Lorusso R, MacLaren G, et al. Initial ELSO Guidance Document: ECMO for COVID-19 Patients with Severe Cardiopulmonary Failure. ASAIO J. 2020 May;66(5):472-4.

6. Clinical management of COVID-19 [Internet]. [cited 2020 Nov 12]. Available from: https://www.who.int/publications-detail-redirect/clinical-management-of-covid-19

7. Alhazzani W, Møller MH, Arabi YM, Loeb M, Gong MN, Fan E, et al. Surviving Sepsis Campaign: guidelines on the management of critically ill adults with Coronavirus Disease 2019 (COVID-19). Intensive Care Med. 2020 Mar 28;1-34.

8. Savarimuthu S, BinSaeid J, Harky A. The role of ECMO in COVID-19: Can it provide rescue therapy in those who are critically ill? Journal of Cardiac Surgery. 2020 Jun 1;35(6):1298-301.

9. Sidebotham D, McGeorge A, McGuinness S, Edwards M, Willcox T, Beca J. Extracorporeal Membrane Oxygenation for Treating Severe Cardiac and Respiratory Disease in Adults: Part 1-Overview of 
Extracorporeal Membrane Oxygenation. Journal of Cardiothoracic and Vascular Anesthesia. 2009 Dec 1;23(6):886-92.

10. Ratnani I, Tuazon D, Zainab A, Uddin F. The Role and Impact of Extracorporeal Membrane Oxygenation in Critical Care. Methodist Debakey Cardiovasc J. 2018;14(2):110-9.

11. Munshi L, Walkey A, Goligher E, Pham T, Uleryk EM, Fan E. Venovenous extracorporeal membrane oxygenation for acute respiratory distress syndrome: a systematic review and meta-analysis. Lancet Respir Med. 2019;7(2):163-72.

12. Brodie D, Bacchetta M. Extracorporeal Membrane Oxygenation for ARDS in Adults. New England Journal of Medicine. 2011 Nov 17;365(20):1905-14.

13. Combes A, Hajage D, Capellier G, Demoule A, Lavoue S, Guervilly C, et al. Extracorporeal Membrane Oxygenation for Severe Acute Respiratory Distress Syndrome. N Engl J Med. 2018 May 24;378(21):1965-75.

14. Schmidt M, Pham T, Arcadipane A, Agerstrand C, Ohshimo S, Pellegrino V, et al. Mechanical Ventilation Management during Extracorporeal Membrane Oxygenation for Acute Respiratory Distress Syndrome. An International Multicenter Prospective Cohort. Am J Respir Crit Care Med. 2019 May 30;200(8):1002-12.

15. Barbaro RP, MacLaren G, Boonstra PS, Iwashyna TJ, Slutsky AS, Fan E, et al. Extracorporeal membrane oxygenation support in COVID-19: an international cohort study of the Extracorporeal Life Support Organization registry. The Lancet. 2020 Oct;396(10257):1071-8.

16. Schmidt M, Hajage D, Lebreton G, Monsel A, Voiriot G, Levy D, et al. Extracorporeal membrane oxygenation for severe acute respiratory distress syndrome associated with COVID-19: a retrospective cohort study. The Lancet Respiratory Medicine. 2020 Nov;8(11):1121-31.

17. Haiduc AA, Alom S, Melamed N, Harky A. Role of extracorporeal membrane oxygenation in COVID-19: A systematic review. Journal of Cardiac Surgery. 2020;35(10):2679-87.

18. Shaheen A, Tanaka D, Cavarocchi NC, Hirose H. Veno-Venous Extracorporeal Membrane Oxygenation (V V ECMO): Indications, Preprocedural Considerations, and Technique. J Card Surg. 2016 Apr;31(4):24852.

19. Hirose H, Pitcher HT, Baram M, Cavarocchi NC. Issues in the Intensive Care Unit for Patients with Extracorporeal Membrane Oxygenation. Crit Care Clin. 2017 Oct;33(4):855-62.

20. RECOVERY Collaborative Group, Horby P, Lim WS, Emberson JR, Mafham M, Bell JL, et al. Dexamethasone in Hospitalized Patients with Covid-19 - Preliminary Report. N Engl J Med. 2020 Jul 17;

21. Guaraldi G, Meschiari M, Cozzi-Lepri A, Milic J, Tonelli R, Menozzi M, et al. Tocilizumab in patients with severe COVID-19: a retrospective cohort study. The Lancet Rheumatology. 2020 Aug 1;2(8):e474-84. 


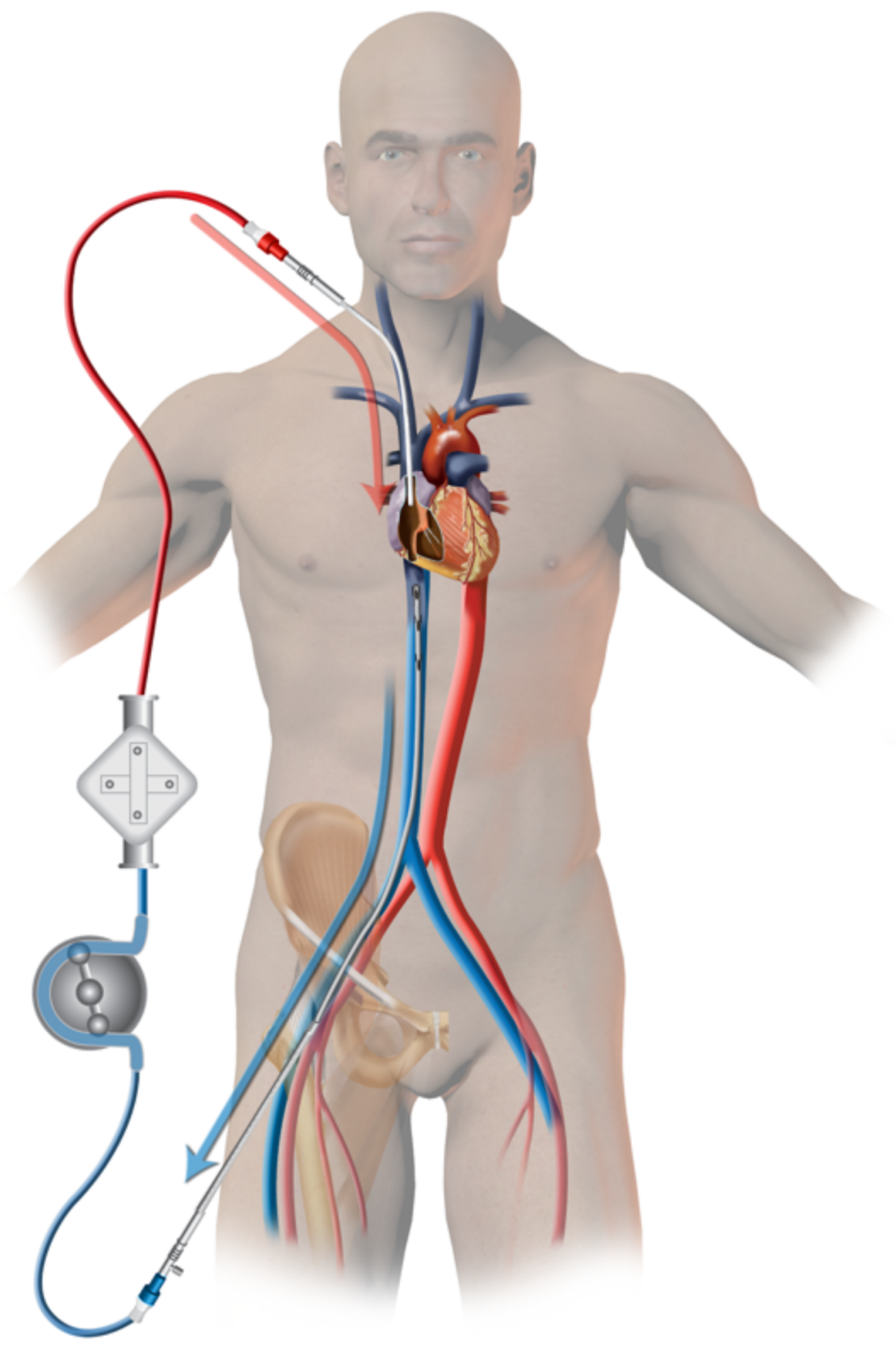

